\title{
Endocrinological Markers for Assessment of Hyperandrogenemia in Hirsute Women
}

\author{
Andreas Mueller ${ }^{\mathrm{a}}$ Susanne Cupisti ${ }^{\mathrm{a}}$ Helge Binder $^{\mathrm{a}}$ Inge Hoffmann ${ }^{\mathrm{a}}$ \\ Franklin Kiesewetter $^{b}$ Matthias W. Beckmann ${ }^{\text {a }}$ Ralf Dittrich ${ }^{\mathrm{a}}$ \\ Departments of ${ }^{\mathrm{a}}$ Obstetrics and Gynecology and ${ }^{\mathrm{b}}$ Dermatology, Erlangen University Hospital, Erlangen, Germany
}

\section{Key Words}

Free testosterone $\cdot$ Bioavailable testosterone $\cdot$ Calculation •

Free androgen index $\cdot$ Hirsutism

\begin{abstract}
Background: Measured endocrinological parameters (total testosterone [TT], free testosterone [FT], dihydrotestosterone [DHT], dehydroepiandrosterone sulfate [DHEAS], and sex hormone binding globulin [SHBG]) and calculated parameters (calculated FT (cFT), calculated bioavailable testosterone (cBT), and the free androgen index [FAI]) in women with hirsutism were compared to the values of a control group. The question remains if cFT or CBT are more appropriate markers for assessment of hyperandrogenemia in clinical situations such as hirsutism in women. Methods: Sixty-six women showed an modified Ferriman-Gallwey (mF-G) score of $\geq 6$ and were classified as hirsutism group and 58 women showed $\mathrm{mF}-\mathrm{G}$ scores of $\leq 5$ and were classified as control group. TT, FT, DHT, DHEAS, and SHBG were measured with enzyme immunoassays in one serum sample. Albumin was measured in the same sample. Afterwards, cFT, cBT, and the FAl were calculated. Results: The hirsutism group revealed significantly increased CFT, CBT, and FAI, while SHBG was significantly decreased compared to the controls. All values of the hirsutism group showed significant, but poor correlation with the mF-G score. SHBG correlated negatively with the $\mathrm{mF}-\mathrm{G}$ score and BMI. Conclusion: Despite the limited correlation of all endocrinological parameters to severity of
\end{abstract}

\section{KARGER}

Fax +4161306 1234 E-Mail karger@karger.ch www.karger.com (c) 2007 S. Karger AG, Basel

0301-0163/07/0671-0035\$23.50/0

Accessible online at:

www.karger.com/hre hirsutism described by the $\mathrm{mF}-\mathrm{G}$ score, $\mathrm{CFT}, \mathrm{CBT}$, and FAl may be more appropriate markers for assessment of hyperandrogenemia in women with hirsutism in comparison with measured values of simple enzyme immunoassays.

Copyright $\odot 2007$ S. Karger AG, Basel

\section{Introduction}

Hirsutism in women is defined as excessive facial and/ or body terminal hairs showing a masculine distribution; the condition affects approximately $7 \%$ of women of reproductive age, and mild forms of hirsutism in particular are the most common [1]. Various scoring systems have been evaluated to quantify hirsutism; the most popular one is a modified version of the Ferriman-Gallwey score (mF-G score), originally proposed in 1961 by Ferriman and Gallwey $[1,2]$. Women with unwanted hair growth should be evaluated endocrinologically, as the majority of these women are found to have an androgen excess (AE) [1]. But it should be noted that not all hirsute women have overt evidence of detectable AE by measuring androgens with routine laboratory methods; subsequently these women are described as suffering from what we understand to be 'idiopathic' hirsutism. Notwithstanding their clinical importance, the prevalence of the different pathological conditions causing or associated with AE remains unclear. The majority of these women may have an $\mathrm{AE}$, but it is unclear which androgen fraction reflects the

Dr. Andreas Mueller

Department of Obstetrics and Gynecology, Erlangen University Hospital

Universitätsstrasse 21-23, DE-91054 Erlangen (Germany)

Tel. +49 9131853 3553, Fax +49 91318533552

E-Mail andreas.mueller@gyn.imed.uni-erlangen.de 
clinical situation most accurately and correlates best with symptoms of hyperandrogenemia such as hirsutism [3].

Testosterone circulates in plasma non-specifically bound to albumin and specifically bound to sex hormone binding globulin (SHBG); a small fraction is unbound as free testosterone (FT). Although immunoassay of blood total testosterone (TT) has long been the standard measurement, the TT concentration is not a reliable index of bioavailable testosterone (BT), as it depends on variations in the concentration of the binding proteins. There is good evidence that the FT and the hormone fraction not specifically bound to albumin in plasma, referred to as the BT fraction, more accurately reflects the clinical situation than total hormone levels in plasma [3]. Several methods of estimating FT and BT in plasma have been established. FT measurement by equilibrium dialysis (apparent FT concentration) $[4,5]$ and centrifugal ultrafiltration $[6,7]$ are the methods of choice for correct measurement of the free fraction of testosterone in vivo. However, this is a time-consuming and complex manual procedure that is not routinely practicable in large laboratories, which rely increasingly on automated multiplex assay platforms.

Alternative methods of estimating FT have therefore been established, using additional assay steps, including the FT analog assay (aFT). aFT measured by an analog ligand immunoassay method is the easiest and fastest, but shows substantially lower values than those obtained by dialysis [8-10]. Models have also been developed for calculating FT (cFT) and BT (cBT) from TT, SHBG, and albumin in one sample $[3,11]$. Another simple calculation model used by many researchers is an indirect parameter of $\mathrm{FT}$, the so-called free androgen index (FAI), which is obtained as the quotient $100 \times \mathrm{TT} / \mathrm{SHBG}$ [12].

Vermeulen et al. presented an excellent evaluation of a calculation method for estimating the FT fraction in serum. They compared apparent FT concentration values, which are generally considered the best method of evaluating FT levels, with cFT and aFT levels as well as with the FAI. Neither aFT nor FAI was a reliable parameter for the FT fraction, but cFT was a reliable index of the FT fraction. The calculated non-specifically bound $\mathrm{T}$ (cBT) reliably reflected non-SHBG-T (BT), and immunoassayable SHBG was a reliable measure of SHBG-binding sites [3].

Souter et al. [1] showed that FT was the most prevalent marker of hyperandrogenemia in 188 women with minimal unwanted hair growth $(\mathrm{mF}-\mathrm{G}$ score of $\leq 5$. But there was no correlation between FT and the $\mathrm{mF}-\mathrm{G}$ score in general and they did not state the method used to esti- mate FT [1]. The question remains if $\mathrm{cFT}$ or $\mathrm{cBT}$ are more appropriate markers for assessment of hyperandrogenemia in clinical situations such as hirsutism in women.

Tsujimura et al. [13] showed that no endocrinological variables differed significantly in patients with partial androgen deficiency of the aging male (PADAM) in Japanese patients. PADAM symptoms correlated neither with measured testosteronelevels nor with calculated testosterone levels.

The aim of the present study was to compare measured laboratory values - TT, aFT, dihydrotestosterone (DHT), dehydroepiandrosterone sulfate (DHEAS), and SHBG and calculated values (cFT, cBT and FAI) in women with $\mathrm{mF}-\mathrm{G}$ score of $\geq 6$ (hirsutism group) to the values of women with $\mathrm{mF}-\mathrm{G}$ score of $\leq 5$ (control group). All values of the hirsutism group were correlated to severity of hirsutism as described by $\mathrm{mF}-\mathrm{G}$ score [14].

\section{Materials and Methods}

\section{Patient Population}

During the study period, 151 women with unwanted facial or body hair growth presented for the evaluation of possible hyperandrogenism at our Division of Gynecological Endocrinology and Reproductive Medicine between January 2004 and January 2005. All women underwent a complete screening panel including physical examination, weight and height measurement, ultrasound examination of the ovary, and further hormonal testing for exclusion of AE related disorder, e.g., polycystic ovary syndrome (PCOS), hyperandrogenic insulin-resistant acanthosis nigricans syndrome (HAIRAN), non-classic adrenal hyperplasia (NCAH), and androgen secreting neoplasm (ASN). The study was approved by the institutional review board at Erlangen University Hospital. All of the patients provided written informed consent and completed a uniform history questionnaire with an emphasis on menstrual dating and regularity, hirsutism and acne, gynecological history, history of infertility, medications, and family history.

\section{Study Design}

Assessment of Hirsutism and Ovulation Status, PCOS and Exclusion of related Disorders. The presence of terminal hair in primarily masculine areas was assessed during the first consultation in every patient by a single investigator. The $\mathrm{mF}-\mathrm{G}$ score was used to describe the hirsutism pattern [14]. The total $\mathrm{mF}-\mathrm{G}$ score represents the sum of the scores for nine body areas. Women with $\mathrm{mF}-\mathrm{G}$ score of 6 or greater were considered to reveal hirsutism (hirsutism group) and women with $\mathrm{mF}-\mathrm{G}$ score of 5 or less were classified as controls (control group) in accordance to Souter et al. $[1,14]$, as shown in figure 1 . The interval between bleeding episodes was assessed. Women with amenorrhea within the previous year were categorized as anovulatory without further testing. In women with regular cycles of between 26 and 30 days, serum was obtained for hormonal analysis between days 3 and 5 of their menstrual cycle, and they were categorized as ovulatory. Women with cycles longer than 30 days, in addition to having an initial 


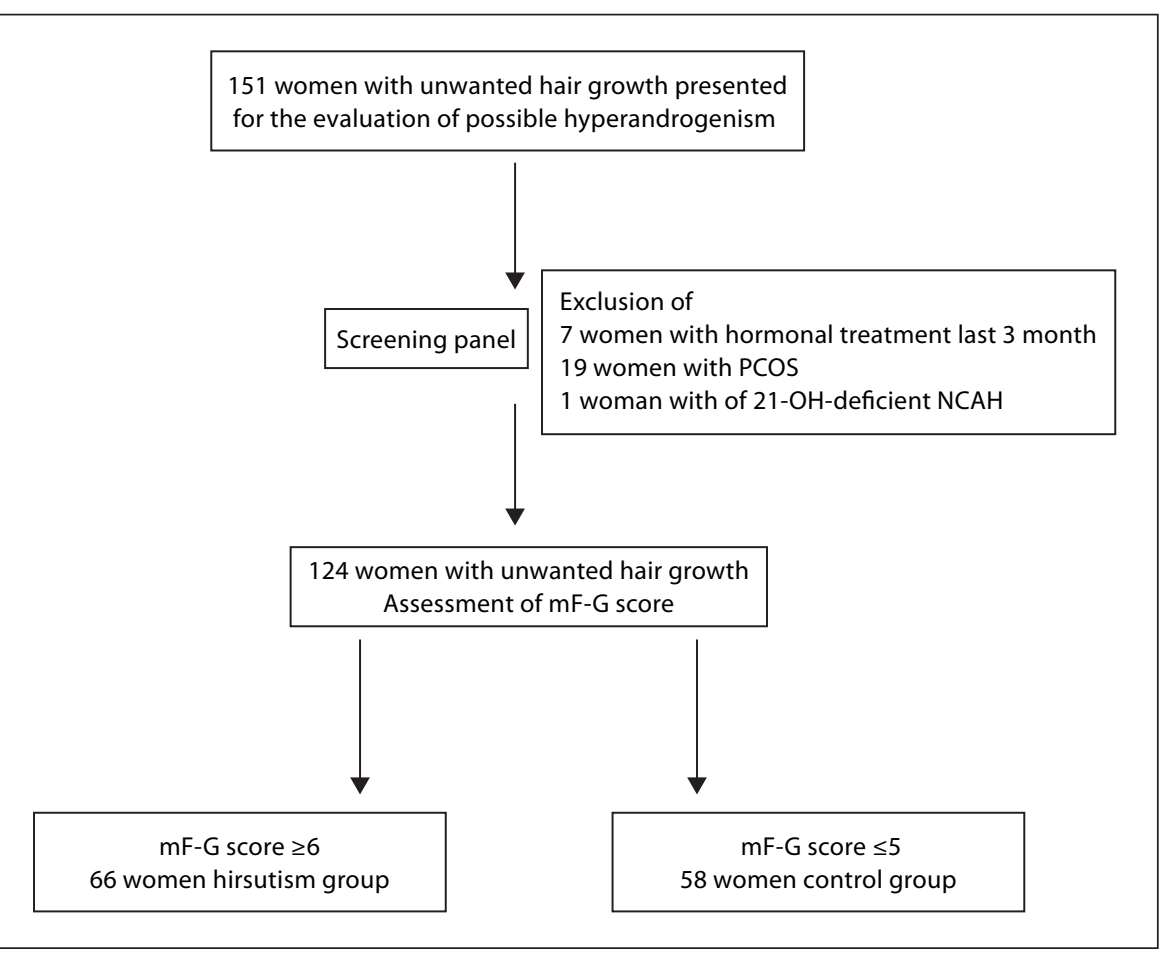

Fig. 1. A flow diagram describing the patient population and the study design.

blood sample taken, had their progesterone level measured on days 22-24 during the same menstrual cycle. If the progesterone level was above $4 \mathrm{ng} / \mathrm{ml}$, the women were considered to be ovulatory [1]. An ultrasound examination of the ovaries was performed and polycystic ovaries were diagnosed in accordance with the diagnostic criteria for PCOS. Women were classified as having PCOS only when they fulfilled the diagnostic criteria for PCOS (two out of three symptoms must be present - revised 2003 diagnostic criteria) [15]. To exclude 21-OH-deficient NCAH patients with repeated 17-hydroxyprogesterone (17-HP) basal levels of $6 \mathrm{nmol} / \mathrm{l}$ or greater, adrenocorticotropic hormone (ACTH) stimulation test was performed and 17-HP levels were measured. A post-ACTH stimulation level of 17-HP to at least $30 \mathrm{nmol} / \mathrm{l} 30$ 60 min after testing was considered diagnostic of $21-\mathrm{OH}$-deficient NCAH [1]. For evaluation for an ASN, our TT cut-off value is above $8 \mathrm{nmol} / \mathrm{l}$, at which we normally carry out computed tomography of the adrenal gland $[1,16]$. These high levels of TT were not found in any of the patients studied.

Women with disorders that result in AE such as PCOS, $\mathrm{NCAH}$, HAIRAN and ASNs and women who had been receiving hormonal therapy within 3 months of their initial visit were not included in the study $[1,16,17]$.

\section{Biochemical Measurements}

Serum was obtained in all patients and immediately assayed for hormonal parameters and albumin in our routine laboratory. All of the assays were carried out in our routine diagnostic endocrinology laboratory using established commercial assays routinely monitored by participation in external quality-control programs. FT was measured using the single-tube Coat-A-Count ${ }^{125}$ I-labeled) FT radioimmunoassay (Diagnostic Products Corp.,
Los Angeles, Calif., USA). The calibration range of the assay was $1.9-173 \mathrm{pmol} / \mathrm{l}$, with an analytical sensitivity of $0.52 \mathrm{pmol} / \mathrm{l}$. The coefficients of variation (CVs) were $18.3,8.5$, and $8 \%$ at the level of $4.1,30.8$, and $69.3 \mathrm{pmol} / \mathrm{l}$. The cross-reaction with $5-\alpha-\mathrm{DHT}$ was $0.041 \%$. DHT was measured with an enzyme immunoassay (Asbach Medical Products, Obrigheim, Germany). The calibration range of the assay was $0-2,500 \mathrm{pg} / \mathrm{ml}$, with an analytical sensitivity of $9.7 \mathrm{pg} / \mathrm{ml}$. The intra-assay $\mathrm{CV}$ was $11.6 \%$, and the inter-assay CV $10.7 \%$ at mid-range concentrations. TT, DHEAS, SHBG were measured with enzyme immunoassays (Immulite 2000, Diagnostic Products Corp., Los Angeles, Calif., USA). The calibration range of the TT assay was $0.7-55 \mathrm{nmol} / \mathrm{l}$, with an analytical sensitivity of $0.5 \mathrm{nmol} / \mathrm{l}$. The cross-reaction with $5-\alpha-\mathrm{DHT}$ was $2 \%$. The calibration range of the DHEAS assay was $0.41-$ $27 \mu \mathrm{mol} / \mathrm{l}$, with an analytical sensitivity of $0.08 \mu \mathrm{mol} / \mathrm{l}$. No crossreactivity with other compounds was known. The calibration range of the SHBG assay was up to $180 \mathrm{nmol} / \mathrm{l}$, with an analytical sensitivity of $0.02 \mathrm{nmol} / \mathrm{l}$. The inter- and intra-assay CVs were always below $11 \%$ at mid-range concentrations. No cross-reactivity with other compounds was known. FAI was obtained as the quotient $100 \times$ T/SHBG. Albumin was regularly measured using routine clinical chemistry methods.

\section{Calculation of Free Testosterone and Bioavailable}

Testosterone

cFT and $\mathrm{cBT}$ were carried out using the formula available on the website of the International Society for the Study of the Aging Male (ISSAM) (http://www.issam.ch/freetesto.htm) from TT, SHBG, and albumin values measured in the same sample from each woman. This method is described in detail by Vermeulen et al. [3]. 
Statistics

All parameters of the Hirsutism-group were compared to the control group using unpaired t test. $p$ values $<0.05$ were considered statistically significant. In the hirsutism group, the correlation between the $\mathrm{mF}-\mathrm{G}$ score and the measured laboratory values and calculated values obtained by the various methods was estimated using the square regression method. In both groups correlations between the SHBG levels and the body mass index (BMI) was estimated using the square regression method. The significance of correlations was estimated by Spearman-Rho analyses. $p$ values $<0.05$ were considered statistically significant. Statistical analysis was carried out using the Statistical Program for the Social Sciences (SPSS, version 10.1 for Windows; SPSS, Inc., Chicago, Ill., USA).

\section{Results}

Seven women were not included as they had been receiving hormonal treatment within 3 months of their initial visit. 19 women were not included as they were diagnosed for PCOS and one woman showed evidence of 21$\mathrm{OH}$-deficient NCAH. All of the women with PCOS had at least one androgen increased. There were no patients with ASNs in our study population. No ovarian tumors were identified using ultrasonography in any of the women and none of the women had a HAIRAN-syndrome. The study population consisted of 124 healthy Caucasian women who presented for the evaluation of their unwanted facial or body hair growth. Sixty-six women with an $\mathrm{mF}-\mathrm{G}$ score of $\geq 6$ were considered to show hirsutism (hirsutism group) and 58 women with an $\mathrm{mF}-\mathrm{G}$ score of $\leq 5$ were classified as controls (control group). The baseline characteristics of both groups are shown in table 1. Both groups showed no significant differences in age or BMI. TT, aFT, DHT, DHEAS, cFT, cBT, FAI, and SHBG of the hirsutism group were compared to the values of the control group. There was no difference measuring albumin, TT, aFT, DHT, and DHEAS between both groups. But cFT, cBT, and FAI were significantly increased and SHBG was significantly decreased in the hirsutism group compared with the control group. Correlation between endocrinological parameters and $\mathrm{mF}-\mathrm{G}$ scores of the hirsutism group are shown in figure 2. TT correlation coefficients of 0.475 , aFT of 0.447 , cFT of $0.429, \mathrm{cBT}$ of 0.461 , and FAI of 0.403 were nearly equivalent. In general, a significant ( $p<0.01$ ), but poor correlation between all of these hormone values and the $\mathrm{mF}-\mathrm{G}$ score was found. SHBG correlated negatively with the $\mathrm{mF}-\mathrm{G}$ score, with a correlation coefficient of -0.246 . Furthermore, SHBG correlated negatively with the BMI, with a correlation coefficient of -0.534 for the hirsutism group and -0.291 for
Table 1. Endocrinological parameters of the hirsutism group compared to the values of the control group

\begin{tabular}{lccc}
\hline & $\begin{array}{l}\text { Hirsutism group } \\
(\mathrm{n}=66)\end{array}$ & $\begin{array}{l}\text { Control group } \\
(\mathrm{n}=58)\end{array}$ & p values \\
\hline Age, years & $30.30 \pm 8.57$ & $32.79 \pm 6.18$ & 0.07 \\
BMI, kg/m & $26.74 \pm 6.91$ & $25.40 \pm 5.26$ & 0.26 \\
mF-G score & $9.96 \pm 3.59$ & $4.10 \pm 0.83$ & $<0.001^{*}$ \\
Albumin, g/l & $46.0 \pm 2.67$ & $46.03 \pm 2.71$ & 0.57 \\
SHBG, nmol/l & $41.16 \pm 15.93$ & $62.96 \pm 38.02$ & $<0.001^{*}$ \\
TT, nmol/l & $2.08 \pm 1.05$ & $1.94 \pm 1.01$ & 0.45 \\
aFT, pmol/l & $7.66 \pm 4.04$ & $6.69 \pm 3.56$ & 0.16 \\
DHT, pg/ml & $421.31 \pm 215.86$ & $396.2 \pm 218.81$ & 0.34 \\
DHEAS, $\mu \mathrm{mol} / 1$ & $5.91 \pm 3.07$ & $5.41 \pm 2.93$ & 0.35 \\
FAI & $6.45 \pm 5.08$ & $4.43 \pm 3.88$ & $0.01^{*}$ \\
cFT, nmol/l & $0.034 \pm 0.022$ & $0.026 \pm 0.018$ & $0.038^{*}$ \\
cBT, nmol/l & $0.88 \pm 0.56$ & $0.67 \pm 0.47$ & $0.029^{*}$ \\
\hline
\end{tabular}

Normal ranges: SHBG: 13-71 nmol/l; TT: 0.52-2.7 nmol/l; aFT: $<8.9$ pmol/l; DHT: 24-363 pg/ml; DHEAS: 0.94-11.67 $\mu \mathrm{mol} / \mathrm{l}$.

* p values $<0.05$ were considered statistically significant.

the control group as shown in figure 3. A significant $(\mathrm{p}<$ 0.01 ) but poor correlation between the SHBG and BMI was found in both groups. The cutaneously generated androgen DHT showed the lowest correlation coefficient with the $\mathrm{mF}-\mathrm{G}$ score at 0.022 .

\section{Discussion}

In this study measured hormone values (TT, aFT, DHT, DHEAS and SHBG) and calculated hormone values (cFT, cBT, and FAI) for assessment of hyperandrogenemia in hirsute women, described by an $\mathrm{mF}-\mathrm{G}$ score of $\geq 6$, were compared to the values of women with an $\mathrm{mF}-\mathrm{G}$ score of $\leq 5$. There is a general consensus that routine endocrine evaluation should be carried out in patients presenting with unwanted hair growth [1]. This study attempted to identify which hormone value is more appropriate for assessment of hyperandrogenemia in women presented with hirsutism. cFT, $\mathrm{cBT}$, and FAI were significantly increased, while SHBG was significantly decreased in the hirsutism group compared to the control group. Therefore $\mathrm{cFT}, \mathrm{cBT}$, and FAI seem to be more appropriate for assessment of hyperandrogenemia in women with hirsutism and an $\mathrm{mF}-\mathrm{G}$ score of $\geq 6$.

Hirsutism may be associated with significant social embarrassment and emotional distress, and it affects approximately $5-15 \%$ of women, depending on racial and 

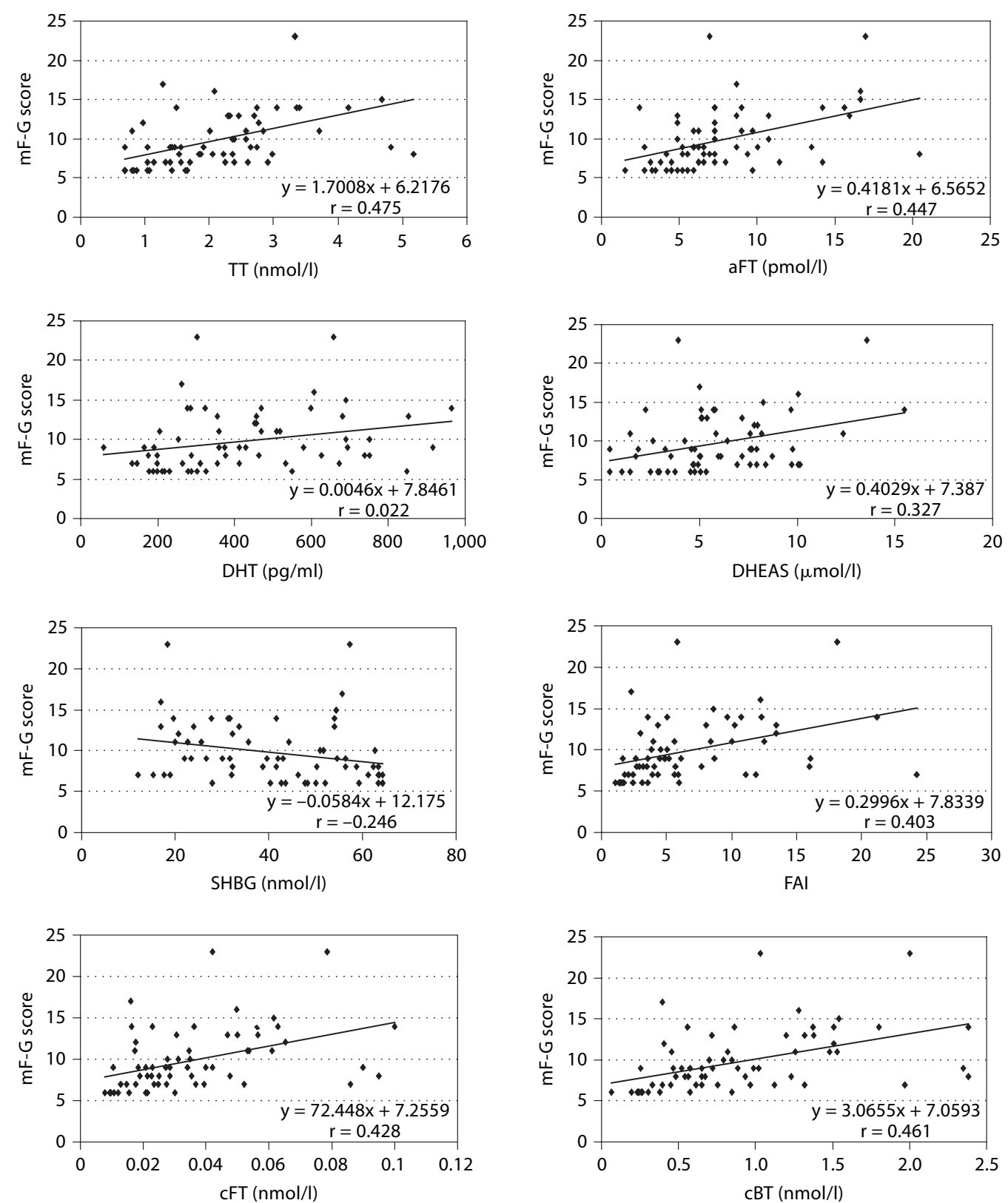

Fig. 2. Comparison of TT, aFT, DHT, DHEAS, SHBG, FAI, cFT and cBT with the clinical situation described by the $\mathrm{mF}-\mathrm{G}$ score in the women of the hirsutism group. A significant $(\mathrm{p}<0.01)$ but poor correlation between all of these hormone values and the mF-G score was found.

ethnic differences. Androgens such as testosterone and DHT irreversibly transform vellus into terminal hair over several growth cycles in areas of the body that are androgen-sensitive. Hair follicles are formed only during fetal development, and the concentration of hair follicles is ge- netically determined $[17,18]$. In general, most women with hirsutism show increases in circulating androgens of unknown causes. Conversely, however, not all of these women have an AE disorder; 5-15\% show normal androgen levels and are described as having idiopathic hirsut- 


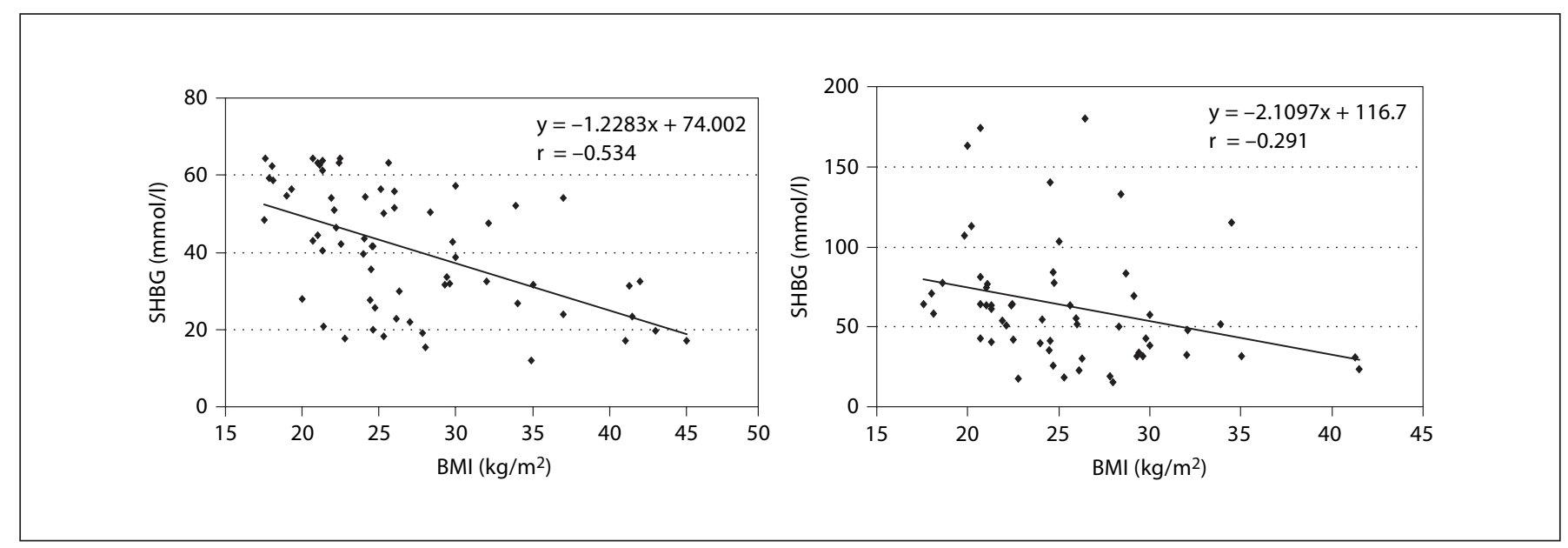

Fig. 3. Comparison of SHBG levels with the BMI in the women of the hirsutism group (left) and in women of the control group (right). A significant $(\mathrm{p}<0.01)$ but poor correlation between the SHBG and BMI was found.

ism' [18]. In addition, the activity of 5- $\alpha$-reductase, which converts testosterone to the more potent DHT is often increased in the hair follicles of hirsute women $[19,20]$.

There has been growing interest in estimating FT in recent years, as the free hormone hypothesis postulates that this small free fraction is the most biologically active fraction and has greater accessibility to tissue [3,21]. The approaches used can be classified into those using simplified laboratory measurements and those based on calculation models using TT and SHBG concentrations (with or without albumin) from the same sample [21]. FT values measured by simplified immunoassay methods do not correlate with the values measured by equilibrium dialysis or centrifugal ultrafiltration, which are the original reference methods for measuring FT in vivo $[3,20]$. Azziz et al. [16] described measured FT as the most prevalent marker, especially in combination with DHEAS, in over 800 patients with AE. Souter et al. [1] described FT as being the most prevalent marker of hyperandrogenemia, elevated in $46 \%$ of all women with minimal unwanted hair growth. Unfortunately, neither of these groups of authors stated the method used to measure FT. The correlation of aFT values with the hirsutism score, with a correlation coefficient of 0.447 in the present study group, was not surprising as the enzyme immunoassay used in this study for measurement of FT showed a good correlation with the golden standard method reported by Van Uytfanghe et al., however measuring only a fraction of the FT concentrations - i.e., 0.20 vs. $2.02 \%$ with FT calculations $[22,23]$.
Vermeulen et al. [3] and Ly and Handelsman [21] reported a better correlation between $\mathrm{cFT}$ values and the FT values measured with equilibrium dialysis or centrifugal ultrafiltration $[3,21]$. On the basis of the free hormone hypothesis that the calculated testosterone parameter more accurately reflects the clinical situation in patients, a better correlation between $\mathrm{cFT}$ or $\mathrm{cBT}$ and the $\mathrm{mF}-\mathrm{G}$ score would have been expected in the present study group. The calculated values should more accurately reflect the clinical situation than total hormone levels or aFT values. However, a closer correlation between the $\mathrm{mF}-\mathrm{G}$ score and the calculated parameters (cFT and cBT) was not found in comparison with those of the measured parameters in this study group. The correlation of the calculated and measured parameters with the mF-G score was equivalent. Tsujimura et al. [13] found also no correlation between PADAM symptoms and any parameter for testosterone levels. They used the same formula at the ISSAM website to calculate $\mathrm{cFT}$ and $\mathrm{cBT}$ in males that was used in the present study. Borgia et al. [24] found also a poor correlation between FT, also measured with an enzyme immunoassay, and the severity of acne in adult women. But there was a strong negative relationship between the severity of acne and SHBG levels. In general, the problem of hirsutism appears to be more related to local processes in the skin that are not adequately recordable by serum testosterone values, regardless whether these are measured or calculated values. Two facts provide support for this hypothesis: firstly, there is free and rapid diffusion of steroids across biomembranes [25]; 
secondly, skin cells express all of the androgen-metabolizing enzymes required for independent cutaneous synthesis of androgens [26]. Furthermore, SHBG levels play an important role in the regulation of $\mathrm{cFT}$ and $\mathrm{cBT}$ and it should be considered that in women, SHBG levels are influenced in a characteristic way by age, obesity and other endocrine disorders, e.g. hyperthyroidism $[27,28]$. In the hirsutism group, we found significant increased cFT and CBT levels which are in part a result of the significant decreased SHBG levels and subsequently this may cause the correlation of low SHBG levels to $\mathrm{mF}-\mathrm{G}$ scores in the hirsutism group.
The result of this study is that there is no closer correlation of the calculated values to the clinical finding, which presents no matter to the hormonal level. But calculated values (cFT, cBT, and FAI) were significantly increased, while SHGB was significantly decreased in hirsute women and seem to be more appropriate to reveal hyperandrogenemia in women with hirsutism.

\section{Acknowledgment}

The study was supported by a grant of the DPC-Akademie, Bad Nauheim, Germany.

\section{References}

1 Souter I, Sanchez LA, Perez M, Bartolucci AA, Azziz R: The prevalence of androgen excess among patients with minimal unwanted hair growth. Am J Obstet Gynecol 2004;191: 1914-1920.

2 Ferriman D, Gallwey JD: Clinical assessment of body hair growth in women. J Clin Endocrinol Metab 1961;21:1440-1447.

3 Vermeulen A, Verdonck L, Kaufmann JM: A critical evaluation of simple methods for the estimation of free testosterone in serum. J Clin Endocrinol Metab 1999;84:3666-3672.

4 Vermeulen A, Stoica T, Verdonck L: The apparent free testosterone concentration, an index of androgenicity. J Clin Endocrinol 1971;33:757-767.

5 McCann DS, Kirkish LS: Evaluation of free testosterone in serum. J Clin Immunoassay 1985;8:234-236.

6 Hammond GL, Nisker JA, Jones LA, Siiteri PK: Estimation of the percentage of free steroid in undiluted serum by centrifugal ultrafiltration-dialysis. J Biol Chem 1980;255: 5023-5026.

7 Vlahos I, Macmahon W, Sgoutas D, Bowers W, Thompson J, Trawick W: An improved ultrafiltration method for determining free testosterone in serum. Clin Chem 1982;28: 2286-2291.

8 Ooi DS, Innanen VT, Wang D, Chong Gl, Donnelly JG, Arseneult JJ, Pronovost C, Wells G: Establishing reference intervals for DPC's free testosterone radioimmunoassay. Clin Biochem 1998;31:15-21.

9 Wilke TJ, Utley DJ: Total testosterone, free androgen index, calculated free testosterone and free testosterone by analog RIA compared in hirsute women and in otherwise normal women with altered binding of sex hormone binding globulin. Clin Chem 1987; 33:1372-1375.

10 Rosner W: Errors in the measurement of plasma free testosterone. J Clin Endocrinol Metab 1997;82:2014-2015.
11 Sodergard R, Backstrom T, Shanbhag V, Carstensen $\mathrm{H}$ : Calculation of free and bound fractions of testosterone and estradiol-17 beta to human plasma proteins at body temperature. J Steroid Biochem 1982;16:801810.

12 Mathur RS, Moody LO, Landgrebbe S, Williamson HO: Plasma androgens and sex hormone binding globulin in the evaluation of hirsute patients. Fertil Steril 1981;35:29-37.

13 Tsujimura A, Matsumiya K, Miyagawa Y, Takao T, Fujita K, Takada S, Koga M, Iwasa A, Takeyama M, Okuyama A: Comparative study on evaluation methods for serum testosterone level for PADAM diagnosis. Int J Impot Res 1981;23:1-5.

14 Hatch R, Rosenfield RL, Moon HK, Tredway D: Hirsutism: implications, etiology and management. Am J Obstet Gynecol 1981; 140:815-830.

15 Rotterdam ESHRE/ASRM-sponsored PCOS Consensus Workshop Group: Revised 2003 consensus on diagnostic criteria and long term health risk related to polycystic ovary syndrome (PCOS). Hum Reprod 2004;19: $1-7$.

16 Azziz R, Sanchez LA, Knochenhauer ES, Moran C, Lazenby J, Stephens KC, Taylor K, Boots LR: Androgen excess in women: experience with over 1,000 consecutive patients. J Clin Endocrinol Metab 2004;89:453-462.

17 Archer JS, Chang RJ: Hirsutism and acne in polycystic ovary syndrome. Best Pract Res Clin Obstet Gynecol 2004;18:737-754.

18 Azziz R: The evaluation and management of hirsutism. Obstet Gynecol 2003;101:9951007.

19 Falsetti L, Gambera A, Andrico S, Sartori E: Acne and hirsutism in polycystic ovary syndrome: clinical, endocrine-metabolic and ultrasonographic differences. Gynecol Endocrinol 2002;16:275-284.

20 Thiboutot D, Chen WC: Update and future of hormonal therapy in acne. Dermatology 2003;206:57-67.
21 Ly PL, Handelsman DJ: Empirical estimation of free testosterone from testosterone and sex hormone-binding globulin immunoassays. Eur J Endocrinol 2005;152:471478.

22 Van Uytfanghe K, Stöckle D, Kaufman JM, Fiers T, De Leenheer A, Thienpont LM: Validation of 5 routine assays for serum free testosterone with a candidate reference measurement procedure based on ultrafiltration and isotope dilution-gas chromatographymass spectrometry. Clin Biochem 2005;38: 253-261.

23 Emadi-Konjin P, Bain J, Bromberg IL: Evaluation of an algorithm for calculation of serum 'bioavailable' testosterone (BAT). Clin Biochem 2003;36:591-596.

24 Borgia F, Cannavo S, Guarneri F, Vannavo SP, Vaccaro M, Guarneri B: Correlation between endocrinological parameters and acne severity in adult women. Acta Dermatol Venerol 2004;84:201-204

25 Oren I, Fleishman SJ, Kessel A, Ben-Tal N: Free diffusion of steroid hormones across biomembranes: a simplex search with implicit solvent model calculations. Biophys J 2004;87:768-779.

26 Zouboulis CC, Degitz K: Androgen action on human skin - from basic research to clinical significance. Exp Dermatol 2004;13:510.

27 Elmlinger MW, Kuhnel W, Wormstall H, Doller PC: Reference intervals for testosterone, androstenedione and SHBG levels in healthy females and males from birth until old age. Clin Lab 2005;51:625-632.

28 Yucel A, Noyan V, Sagsoz N: The association of serum androgens and insulin resistance with fat distribution in polycystic ovary syndrome. Eur J Obstet Gynecol Reprod Biol DOI:10.1016/j.ejogrb.2005.11.012. 